\title{
DES MEULES NEUVES POUR UN PAIN MEILLEUR? UN FORGERON PERPIGNANAIS ET SON COMMERCE DE MEULES
} (1410-1422)

\author{
DO NEW MILLSTONES MAKE BETTER BREAD? \\ A BLACKSMITH FROM PERPIGNAN AND HIS MILLSTONE TRADE, 1410-1422
}

Aymat CATAFAU

Université de Perpignan - Cresem (Axe Patrimoines) catafau@univ-perp.fr

\begin{abstract}
Resumen: Joan Bastard était un forgeron établi à Perpignan au début du $\mathrm{XV}^{\mathrm{e}}$ siècle. Toutefois, il semble avoir été avant tout un marchand de meules. Entre 1410 et 1422, il vendit en effet plus de 100 meules en Roussillon, Vallespir et Bas-Conflent. Les documents notariés relatifs à ce commerce sont d'une qualité exceptionnelle. Ils permettent non seulement d'étudier un système commercial particulier mais aussi de mieux connaître les meules en usage sur le versant septentrional des Pyrénées Orientales. Joan Bastard vendait ses meulesà crédit.Il offrait principalement des meules importées, taillées dans de la « pierre de Montjuïc », un grès qui rendait possible l'obtention d'une farine plus fine que celles obtenues avec les meules de grès ou de granites locaux.
\end{abstract}

Mots-clés: meules, grès (pierre), commerce, Roussillon médiéval, farine, $\mathrm{XV}^{\mathrm{e}}$ siècle.

\begin{abstract}
Joan Bastard was a blacksmith established in Perpignan in the early $15^{\text {th }}$ century. Nevertheless, he seems to have developed his main professional activity as a millstone trader. Between 1410 and 1422, he sold more than 100 millstones in Roussillon, Vallespir and BasConflent. Notarized documents related with this trade are of an exceptional quality. They allow not only to study a particular commercial system but also to know the millstone in use in the north side of Oriental Pyrenees. Joan Bastard sold his millstones at credit. He offered almost imported millstones of "Montjuïc stone", a sandstone that made possible to obtain a flour finer than ones produced using millstones of local sandstone and granites.
\end{abstract}

Key words: millstone, sandstone, trade, medieval Roussillon, flour, $15^{\text {th }}$ century. 


\section{Introduction}

À Juan Utrilla Utrilla, une affection immédiate m'a uni dès nos premières rencontres. Au cours des travaux de notre groupe RESOPYR, ce sentiment n'a cessé de s'enrichir de toutes nos discussions. Le respect pour le grand chercheur s'est ainsi ajouté à l'estime pour l'homme élégant, chaleureux et courtois, forgeant une amitié, qui, malgré l'éloignement, demeure ferme et qui m'est précieuse.

J'avais « sous le boisseau », depuis six ans, dans une « chemise » toujours posée sur mon bureau, un beau dossier de documents mis de côté pour une petite tentative de micro-histoire économique que je me promettais de faire, à l'occasion, quand le temps me le permettrait. Les hommages à Juan et la réclusion contrainte du printemps 2020 m'ont donné le prétexte et le temps de dépoussiérer ce paquet de notes. J'ajouterai que, comme beaucoup de " confinés ", je me suis essayé à pétrir moi-même mon pain durant ces semaines, et, si je n'ai pas percé tous les mystères de la panification, le choix des diverses variétés de farine m'a beaucoup occupé, et m'a sans doute rendu réceptif à certains points de vue abordés ici. J'avais eu d'emblée le sentiment de tenir avec ce dossier de documents le matériau d'une petite chronique de l'activité d'un forgeron, spécialisé dans la vente de meules de moulins, qui pouvait être amusante à reconstituer, et peut-être intéressante à lire. J'espère que Juan trouvera, parmi ces meules et ces céréales, un peu de notre Roussillon médiéval et un gage de mon amitié.

Préparant un colloque qui devait commémorer la rencontre de 1415 à Perpignan, ${ }^{1} \mathrm{j}$ 'avais décidé de lire tous les registres de notaires conservés de cette année-là pour la ville et le Roussillon. Il me semblait que l'arrivée de plusieurs milliers de nobles, clercs, cavaliers, serviteurs devait avoir produit une activité particulière chez les notaires, ce que la lecture de ces registres m'a confirmé.

Lors du dépouillement du registre du notaire Pere Baseli pour l'année 1415 je prenais note, au fil des pages, des informations m'intéressant à divers titres. Comme j'avais suivi et accompagné les travaux de Michel Martzluff sur les

1 Aymat Catafau, Nikolas Jaspert, Thomas Wetzstein, Perpignan 1415. Un sommet européen à l'époque du Grand Schisme d'Occident, Zurich, LIT Verlag, 2018, $677 \mathrm{p}$. 
meules de Vilarnau, ${ }^{2}$ puis sur celles du massif de Rodès, ${ }^{3}$ la mention de la vente d'une meule faisait partie de ce que je notais systématiquement. Elles sont d'ailleurs assez rares pour être remarquées. Au bout du registre de Baseli j'avais noté dix actes de vente de meules enregistrés pour cette seule année, un chiffre extraordinaire, d'autant que c'était toujours le même homme qui les vendait, un nommé Joan Bastard, forgeron de Perpignan.

\section{Joan Bastard, forgeron de Perpignan, marchand de meules}

Une curiosité de plus s'ajoutait aux actes de ventes de meules. En marge de la page du registre, chacune des ventes de meules par Joan Bastard était indiquée d'un signe tracé à la plume par le notaire, un rond marqué d'un point central, comme la schématisation d'une meule : $\odot$ Ce signe me permit, quand je l'eus compris, de retrouver toutes les ventes de meules faites par Joan Bastard que P. Baseli avait enregistrées cette année-là.

Il semblait que Joan Bastard ait choisi Pere Baseli pour notaire, en passant ses actes chez lui. Une exploration parmi les volumes de ce notaire conservés et consultables m'a permis de retrouver Joan Bastard dans les registres des années 1410 à $1422,{ }^{4}$ toujours avec de nombreuses ventes de meules, chaque fois signalées en marge du même symbole. Les autres actes enregistrés pour ce forgeron, de caractère professionnel ou familial, ne comportaient pas ce symbole. Mais le forgeron passait aussi des actes, y compris des ventes de meules, chez d'autres notaires. Il m'a été possible d'en retrouver un certain nombre.

2 Michel Martzluff et alii, «Meules et moulins de Vilarnau», dans Olivier Passarrius, Richard Donat et Aymat Catafau (dir.), Vilarnau. Un village du Moyen Âge en Roussillon, Perpignan, Trabucaire, 2008, p. 314-384.

3 Michel Martzluff, «Les techniques primitives : extraction et façonnage opportunistes des meules en granit», dans Olivier Passarrius, Aymat Catafau et Michel Martzluff, (dir.), Archéologie d'une montagne brûlée, Perpignan, Trabucaire, 2009, p. 315 et suivantes.

4 Consultés aux Archives Départementales des Pyrénées-Orientales, série 3E1, registres de Pere Baseli : $\mathrm{n}^{\circ} 829$ (année 1410, manuel incomplet), 1432 (1407, manuel), 1434 (1420, manuel), 1713 (1406, manuel), 1716 (1409, notule), 1717 (1410 notule et fragment de manuel), 1721 (1413, manuel), 1723 (1415, manuel), 1725 (1417, manuel), 1726 (1418, manuel), 1727 (1419, notule), 1729 (1421, manuel), 1730 (1423, notule), 1732 (1412, manuel), 1737 (1434 manuel). Plusieurs de ces manuels, en état de conservation médiocre, m'ont été communiqués à titre exceptionnel, grâce à l'amabilité de M. Denis Fontaine, des ADPO, qui les a mis en condition de pouvoir être manipulés sans dommages, un travail dont je le remercie grandement. Plusieurs des importantes informations que j'ai relevées seraient resté inconnues sans cet effort. 
Je ne prétends pas avoir dépouillé l'ensemble des registres notariaux qui pourraient contenir des informations à propos de Joan Bastard, mais il me semble que plus de cent de documents réunis permettent d'ouvrir une fenêtre sur la vie et les activités d'un artisan qui se double d'un importateur et marchand d'un produit très spécifique. Je n'aborderai ici que ce commerce de meules, le point le plus remarquable et le plus original de l'activité de Joan Bastard, et j'essaierai d'en proposer une interprétation.

J'ai dit que les mentions de ventes de meules ne sont pas très fréquentes. Elles sont plutôt rares au XIV $\mathrm{XI}^{\mathrm{e}}$ siècle, et toujours très ponctuelles. Pour ce siècle, Sylvie Caucanas ${ }^{5}$ relève une quinzaine de mentions seulement d'extraction et de vente de meules : l'extraction des meules à la meulière du Boulou (lieu-dit els Molars) se fait sous seigneurie royale en 1319, le procureur royal y achète d'ailleurs 8 meules portées au moulin neuf de Salses reconstruit en 1353, mais on trouve dès 1345 l'importation de meules pour les moulins de Rivesaltes déchargées sur la plage de Canet, et de trois meules de moulin en pierre de Barcelone en 1378 et 1391. D'ailleurs en 1393 est créée une société entre trois hommes : un moler barcelonais, un pareur et un meunier perpignanais, pour importer en Roussillon des meules de Barcelone, pour lesquelles le moler doit avoir un droit d'exploitation.

Bien qu'un peu plus fréquents au $\mathrm{XV}^{\mathrm{e}}$ siècle, les actes de vente de meules restent rares. La série que nous présentons ici est, dans les archives des Comtés nord-catalans, vraiment exceptionnelle. Avant d'essayer de comprendre pourquoi ces ventes de meules sont si nombreuses et ce que l'on peut déduire de ce commerce, examinons d'abord la nature et l'ampleur de cette documentation.

\section{Les ventes de meules par Joan Bastard : nombre et chronologie}

Entre 1410 et 1422, nous avons pu comptabiliser 77 actes se rapportant à des ventes de meules par Joan Bastard, pour un total de 105 meules vendues. Voilà quelle en est la distribution par années et par notaires :

5 Voir Sylvie Caucanas, Moulins et irrigation en Roussillon du IXe au XVe siècle, Paris, CNRS Histoire, 1995, p. 148-149 et notes 36 à 40, pour les références de tout ce paragraphe. Interrogée récemment, Sylvie Caucanas m'a indiqué, que, autant qu'elle puisse l'affirmer avec prudence, peu d'actes de ventes de meules du XIV ${ }^{\mathrm{e}}$ siècle ont dû échapper à son dépouillement. 


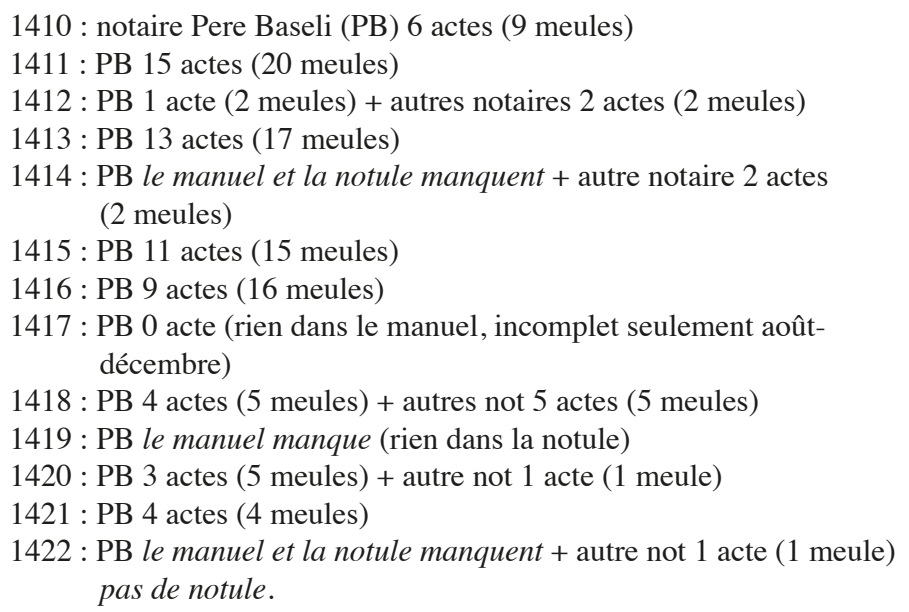

Il est clair que la relation entre Joan Bastard et le notaire Baseli est étroite, et que Joan Bastard choisit préférentiellement ce notaire pour passer ses actes, y compris les actes autres que ceux concernant les meules. C'est aussi chez lui que le frère Joan, Jaume, et son épouse Catherina enregistrent leurs actes.

Les manuels de Pere Baseli conservés et antérieurs à 1410 (seulement 1406 et 1407), ne comportent aucun acte de vente de meule par Joan Bastard, et aucun acte le concernant, même si son frère Jaume Bastard, apparait trois fois en 1406. Dans le manuel de 1437 de Pere Baseli, Joan Bastard est absent, tout comme sa famille.

Cinq manuels de Baseli (pour les années 1410, 1411, 1413, 1415, 1416) donnent à eux seuls un total de 54 actes pour 77 meules (auxquels on doit ajouter 4 actes de vente pour 4 meules chez d'autres notaires ces années-là). Ce sont là les indicateurs d'une activité surprenante, vraiment intense, qui apparaît subitement et ne se poursuit pas sur le même rythme. L'anomalie dans les sept années 1410-1416, est l'année 1412, pour laquelle, dans le manuel conservé de P. Baseli un seul acte concerne une vente de deux meules et nous avons pu rencontrer chez deux autres notaires pour la même année deux autres actes, qui ne donnent qu'un total de 4 meules vendues en 1412. Pour l'année 1414 le manuel de P. Baseli manque, et il est impossible de tirer un indice des deux actes conservés par un autre notaire. Que se passe-t-il en 1412 pour Joan Bastard ? La chute des ventes est-elle due à des problèmes d'approvisionnement en meules ? En tout cas la demande reste présente, comme le prouvent les chiffres de 1413 puis de 1415 et 1416 . 
Pour expliquer cette soudaine multiplication du commerce de meules, il convient de faire appel à deux éléments d'explication : la stratégie de vente elle-même, fondée sur le crédit, et le produit vendu, dont la demande devient généralisée.

\subsection{La vente à crédit des meules}

Les actes concernant les meules sont très normalisés. Ils sont tous, sans exception aucune, des actes de reconnaissance de dette : un homme (le plus souvent un meunier) déclare devoir une somme pour l'achat d'une ou quelquefois plusieurs meules, d'une dimension de 5 palms (rarement 5 palms et demi), à Joan Bastard, forgeron de Perpignan, présent à l'acte (parfois absent, représenté en ce cas par le notaire). Puis viennent les indications sur les modalités de paiement ou la date d'échéance du prêt, suivies des formules juridiques de rétorsion en cas de non paiement. Rarement le débiteur donne un garant (fidéjusseur) qui ajoute une phrase d'acceptation à la fin de l'acte. Viennent les témoins (deux en général, plus le notaire).

La plupart de ces reconnaissances de dettes font l'objet d'une annulation, d'une quittance inscrite : la notice de cancellation indique qu'elle a été faite sur mandat de Joan Bastard ou de son procureur (Pere Alissen hôtelier et beaufrère de Joan Bastard ou Pere Alissen prêtre et neveu de Joan Bastard ou Jaume Bastard, frère de Joan Bastard) puis deux témoins et le notaire.

Un point à souligner donc est la forme financière que prend ce négoce de meules : la vente à crédit. Certes Sylvie Caucanas a relevé dans sa thèse une mention de vente directe de meule, au comptant, ${ }^{6}$ ce qui permet de supposer que cette modalité de vente peut faire aussi l'objet de la rédaction d'un acte notarié. Cependant on conçoit qu'une négociation directe d'un bien meuble, avec paiement et «emportement » immédiats, est moins nécessairement enregistrée chez le notaire, car cet enregistrement a un coût. On dira que les ventes de chevaux, d'animaux en général, ou celles d'esclaves donnent lieu à la rédaction d'actes sans doute quasi systématiques. Mais ces animaux ou esclaves sont susceptibles de contestation ou de revente, d'où la nécessité de pouvoir faire preuve de l'achat et de sa date.

6 S. Caucanas, Moulins et irrigation en Roussillon..., p. 157, note 40. De ces quatre mentions de vente directe, avec paiement au comptant, trois sont issues de registres de comptabilité royale, qui doivent enregistrer toutes les dépenses. Une seule est une vente enregistrée devant notaire. 
Quoi qu'il en soit, nous n'avons pas trouvé d'acte de vente directe de meule par Joan Bastard, et les deux autres (seulement deux !) actes de ventes de meules par d'autres vendeurs, en 1424 et 1442, sont aussi des ventes à crédit. La vente à crédit, même pour des sommes bien inférieures au prix d'une meule, génère le plus souvent un acte de reconnaissance de dette. Si Joan Bastard vend des meules au comptant, nous n'en avons pas trace, mais il vend tant de meules à crédit que nous pouvons en déduire qu'il s'agit là de son procédé de vente préféré, peut-être même exclusif. Il n'est pas douteux que l'offre systématique du crédit puisse expliquer le succès de sa stratégie de vente.

En revanche, Joan Bastard est attentif au paiement. Sur 77 actes de reconnaissance de dette, 16 seulement ne portent pas la notice de cancellation attestant de leur règlement, encore cela ne veut-il pas dire que les débiteurs n'aient pas réglé leur dette à l'occasion d'une autre transaction, par exemple lors d'une vente de céréales. Il arrive que ces quittances portent une date très tardive, deux ou trois années après la reconnaissance, une fois même cinq ans après (dette du 25 septembre 1421, cancellée le 20 octobre 1426). ${ }^{7}$ Joan Bastard n'est d'ailleurs pas un créditeur oublieux ou laxiste. Le 26 août 1421 son mandataire fait mettre en prison, jusqu'au moment où il aura payé sa dette, un tisserand qui lui doit le prix d'une meule acquise le 6 juin de l'année précédente. $^{8}$

Les reconnaissances de dette pour vente de meules enregistrées par le notaire Pere Baseli, entre 1411 et 1420 présentent toutes, sans exception, le même signe en marge, ce rond marqué d'un point central qui peut symboliser une meule. Pere Baseli a utilisé d'autres signes (des croix particularisées) pour identifier dans ses registres et retrouver facilement des actes concernant certains établissements religieux; cela reste exceptionnel et limité à quelques actes par registre. Pour certains d'entre eux il note le sens de ces signes sur la page de garde de son registre. Nous n'avons pas trouvé d'explication au rond pointé au centre, mais son rapport avec les meules est évident : seuls les actes concernant une ou plusieurs meules en sont signalés, les autres actes concernant

7 ADPO, 3E1/1729, fol. $62 v^{\circ}$. Cette date est importante, elle est la mention la plus tardive de Joan Bastard que nous ayons conservée.

8 Acte enregistré par le notaire Pere Vila de Perpignan, mention dans 3E1/1729 inter fol 57, 26/08/1421 Baseli : action de Pere Alissend clerc, procureur de Joan Bastard devant le juge de la cour du bayle de Perpignan pour paiement de dette contre Georgius Hugueti textor Perpiniani; au verso : Jordi Huguet a été mis en prison jusqu'à ce qu'il paie. 
Joan Bastard ne portent pas cette marque. Soit le notaire a marqué ces actes au fur et à mesure de leur rédaction, soit ils ont été recherchés et pointés après coup, à un moment où Baseli a eu besoin, ou mandat, de le faire. L'explication d'un tel inventaire de ces actes précis pourrait résider dans une demande de Bastard : « rechercher et comptabiliser toutes les meules vendues par moi »... la raison ne peut en être qu'économique. Les meules importées paient une taxe à leur arrivée au port, mais pas au moment de leur vente, il ne s'agit pas d'une 'inspection fiscale' d'un type inédit, et inconnu à l'époque. Le plus probable est que Joan Bastard est associé dans une compagnie d'importation, comme celle dont Sylvie Caucanas a trouvé trace en 1393 entre un pareur et un meunier de Perpignan et un faiseur de meules ('meulier') de Barcelone pour l'importation de meules de cette ville à Perpignan. ${ }^{9}$ Au moment de liquider une association de ce type, ou pour une reddition de comptes finale, le notaire remet peut-être à Joan Bastard un décompte détaillé du nombre de meules et des montants enregistrés. Même les reconnaissances de dette non cancellées (non remboursées ?) sont ainsi pointées, ce qui suppose une comptabilité complexe.

Nous savons par un acte de juillet 1413 qu'un brassier, un homme de condition modeste donc, reconnaît devoir à Joan Bastard une somme assez faible, $15 £$ (le prix de deux ou trois meules, si cela peut être notre unité d'évaluation), qui représente la part de bénéfices de Joan Bastard dans une société qu'ils avaient en commun, à laquelle s'ajoute une dette pour un prêt consenti par Joan Bastard. Un acte enregistrant cette société a été dressé chez le même notaire qui enregistre l'acte, acte que nous n'avons pas retrouvé. Le montant dû, comme le rang social de l'associé, semblent toutefois trop modestes pour concerner l'important commerce de meules dont il s'agit ici.

\subsection{Les meules, prix, dimension, matériau, provenance}

Au cœur de ce négoce, figurent les meules. Elles sont appelées mola, ou rota lapidea : en effet l'appareillage du moulin comporte aussi une roue de bois, que l'on achète, ${ }^{10}$ la précision 'roue en pierre' n'est donc pas un pléonasme. Le notaire ajoute le plus souvent molendini bladerii, exprimé une fois en catalan de molí blader. 
Toutes les meules vendues ${ }^{11}$ ont une taille ${ }^{12}$ de 5 palms. Le palm ou pam représente $1 / 8^{\mathrm{e}}$ de la canne qui, en Roussillon, est celle de Montpellier, de 1,98 $\mathrm{m}$. Le palm étant donc d'environ 0,25 $\mathrm{m}$, le diamètre des meules est donc de $1,25 \mathrm{~m}$. Seules quatre sont d'un diamètre un peu supérieur, de 5 palms et demi, soit 1,37 m (Caucanas, 1995 ; Martzluff, 2008 : 336).

Ces dimensions correspondent à celles mentionnées dans les actes du XIV ${ }^{\mathrm{e}}$ siècle retrouvés par $\mathrm{S}$. Caucanas ; leur gabarit est adapté à la force motrice des moulins hydrauliques qui depuis le milieu du XII siècle font tourner des roues supérieures à 1 mètre de diamètre, qui semblait le gabarit des moulins des IX $\mathrm{C}^{\mathrm{e}}$ $\mathrm{XII}^{\mathrm{e}}$ siècles qui étaient peut-être plus souvent à roue mobile verticale, plus petite, inférieure à 1 mètre de diamètre. ${ }^{13}$

Le prix de ces meules varie autour d'une valeur médiane de $6 £ 1 \mathrm{~s}$. La moins chère a été vendue pour $4 £ 10 \mathrm{~s} 6 \mathrm{~d}$, la plus chère pour $7 £ 14 \mathrm{~s}$. Une variation de prix importante, pour laquelle nos documents permettent de suggérer deux explications :

1) D'abord la qualité de la meule, chacune des meules est vendue 'telle qu'elle est' (talis qualis est).${ }^{14}$ Plusieurs éléments matériels peuvent en effet intervenir dans la variabilité de prix. La qualité de la pierre n'est pas directement en cause, car la provenance des meules est sans doute unique, on le verra, mais l'épaisseur de la meule, la présence d'éclats ou de microfailles apparues par exemple au percement de l'œil, ou de chocs intervenus lors du transport, peuvent expliquer une variation de prix, autour d'un prix moyen de $6 £$ environ.

2) Au-delà de la qualité de la meule, le prix inclut aussi une part pour le transport. Ainsi, un document précis nous indique que le prix d'une meule vendue par Joan Bastard ne sera pas le même si l'acheteur la prend à Perpignan, ou va la chercher directement à Collioure, qui est le port de débarquement. Des meules prises à Collioure sont vendues $5 £ 10$ s chacune, en 1411 et en 1415. En 1412 une vente comportait même la disposition suivante : « Il est convenu entre nous que si vous prenez [cette meule] de celles que j'ai à

11 Sauf quatre dont le notaire Pere Baseli a omis la mesure.

12 Le notaire utilise l'expression de rana pour indiquer la dimension.

13 Michel Martzluff et alii, « Meules et moulins de Vilarnau... », p. 339, et Michel Martzluff, " Les techniques primitives... », p. 319.

14 La même expression est employée pour les ventes de bétail, d'animaux de trait, de selle ou de bât et d'esclaves, où les qualités physiques peuvent être la raison de variations. 
Perpignan, vous la paierez 11 florins et demi (le florin vaut 11 sous : soit $6 £ 6 s$ et $6 \mathrm{~d}$ ) et si vous la prenez de celles que j'ai à Collioure, vous la paierez 10 florins $(5 £ 10 \mathrm{~s}) »^{15} \mathrm{Il}$ est possible que les montants des dettes incluent donc une part de surcoût pour le transport.

La qualité de l'objet vendu, le produit, la meule, est sans doute un élément expliquant le succès commercial de Joan Bastard. La quasi totalité des actes inscrits par Baseli ne renseignent que sur le diamètre des meules vendues, fort heureusement un acte enregistré par un autre notaire, en $1422,{ }^{16}$ apporte un renseignement essentiel : la meule vendue vient de Barcelone et précisément est en « pierre de Montjuïc ». Cette information peut être étendue à l'ensemble des meules vendues par Joan Bastard ainsi que l'indiquent plusieurs actes mentionnant l'enlèvement des meules au port de Collioure à prix réduit, donc leur arrivée par bateau. La " pierre de Montjuïc » de Barcelone ${ }^{17}$ est un grès dont certaines variétés ont des qualités particulières qui rendent ces meules plus résistantes à l'usure. ${ }^{18}$

15 Est tamen conventum inter nos quod si vos recipitis de molis quas ego habeo infra villam Perpiniani, quod solvatis pro eadem dictos XI florenos et medium, si tamen recipitis de molis quas ego habeo intus villam Cauquoliberi solvatis pro eadem decem florenos tamen, 3E1/1014, fol. 7r, notaire Gabriel Resplant, a. 1412. Comme on le verra pour les informations sur le matériau et la provenance, c'est un notaire différent de Pere Baseli qui rédige des actes plus complets, plus explicites. Les actes de enregistrés par Pere Baseli pour Joan Bastard sont extrêmement normalisés, quasiment interchangeables pour leur contenu, et réduits au strict minimum, comme pour réduire aussi le coût de rédaction des actes.

16 Nos Jacobus Breto monerius loci de Rayneriis et Petrus Pages monerius loci de Bossachs ambo insimul [...] confitemur nos debere vobis Johannis Bastard fabro Perpiniani presenti novem florenos et medium auri de Aragonia quorum precio nobis vendidistis unam molam de pera de Mont Juhic de Barchinona, rane quinque palmorum [...], 3E1/1531, 46v, Jordi Masdamont.

17 Mon collègue Michel Martzluff, comparant le grès de la carrière de Molars, près du Boulou, qui a servi à fabriquer des meules depuis l'époque tardo-antique (meules d'un diamètre très inférieur à un mètre), à celui de Montjuïc de Barcelone, observe : « Notons donc qu'il existe sur le Montjuïc de Barcelone plusieurs types de grès. Le plus banal est à ciment calcaire : c'est principalement celui qui est utilisé dans la construction de cette ville. Il est plus facile à travailler, plus tendre et joliment coloré en ocre. La qualité supérieure se retrouve dans certains bancs médians des falaises de Montjuïc et se localise dans une carrière surtout exploitée en meulière au Moyen Âge. Ce grès « acide », comme celui du Boulou, possède un ciment siliceux qui le rend très solide et propice pour fabriquer les meules et des éléments porteurs du bâti. [...] Le grès du Boulou comprend plus de grains schisteux et surtout des cristallisations secondaires de silice qui sont engrenées autour des fragments sableux et ménagent des vides. Le ciment siliceux des grès de Montjuïc, parfois de l'opale, remplit tous les vides comme dans un quartzite. » (Je le remercie pour ces remarques, qui donnent des éléments concrets indispensables à la compréhension de ce commerce).

18 Douze meules taillées dans la pierre de Montjuïc sont, dès 1331, importées à Castelló d'Empúries pour les nouveaux moulins construits sous l'autorité comtale. L'achat et le transport de ces meules coûta $315 \mathrm{~s} 6 \mathrm{~d}$, soit un prix moyen de $1 £ 6 \mathrm{~s} 3 \mathrm{~d}$ et 1 obole par meule, à savoir $216 \mathrm{~s}$ pour l'achat des meules (18s par meule), 12s $6 \mathrm{~d}$ pour le transport de Montjuïc à la mer et pour les taxes perçues 


\section{Joan Bastard, un commerçant ingénieux, pour un marché nouveau?}

Relions ensemble les éléments dont nous disposons :

- plus de 100 meules de moulins vendues en 13 années par Joan Bastard, en Roussillon, Vallespir et dans le bas Conflent. Ceci n'est qu'une partie de ses ventes, car plusieurs registres du notaire 'particulier' de Joan Bastard nous manquent, et l'on a pu constater que certaines de ses ventes sont enregistrées par d'autres notaires, que nous n'avons pas systématiquement dépouillés pour cette décennie ;

- un système commercial qui est exclusivement fondé sur la vente à crédit;

- la fourniture de meules importées, d'une pierre de haute qualité, supérieure à toutes celles produites en Roussillon.

Il faut s'interroger sur les raisons de ce commerce. La vente à crédit et la monopolisation du commerce des meules importées de Barcelone par Joan Bastard lui permettent de répondre à une demande devenue générale. Elle repose sur le désir des meuniers de s'équiper en meules de pierre de Montjuïc.

En effet les meules faites cette pierre sont plus résistantes que celles en grès du Boulou, ${ }^{19}$ traditionnellement produites en Roussillon. Pour une bonne partie, la production de meules dans notre région est alimentée jusqu'au XIX ${ }^{\mathrm{e}}$ siècle par les carrières de meules de granite de Reglella et Casesnoves (commune d'Ille-sur-Têt) ou de Rodès, ${ }^{20}$ qui sont sans doute

à Barcelone, et $87 \mathrm{~s}$ pour le transport par navire de Barcelone à Castelló (Josep Maria Gironella $\mathrm{i}$ Granés, Els molins i les salines de Castelló d'Empúries al segle XIV, Barcelona, Fundació Noguera, 2010 , p. 215.). L'auteur souligne que la grande majorité des meules utilisées dans les moulins comtaux de Castelló proviennent des carrières voisines de Peratallada et de Canapost. De fait, la riche documentation de Castelló donne mention de beaucoup de meules achetées dans les carrières locales, mais les seules achetées à Barcelone, en pierre de Montjuïc, sont celles qui équipèrent les moulins comtaux au moment de leur construction, comme pour une dépense de prestige. Ceci confirme que les meules de Monjuïc, exploitées et exportées jusqu'au comté d'Empúries, restent un produit d'exception.

19 À propos du grès du Boulou et des inconvénients de cette variété de pierre meulière, Michel Martzluff indique : "Mais ce grès local, surtout le grès conglomératique exploité sur le haut des carrières jusqu'au XIV ${ }^{\mathrm{e}}$ siècle (grandes meules de 1,16 à 1,30 cm) comprend des galets et fragments de schiste paléozoïque assez nombreux, des gneiss et granites altérés qui sont des faiblesses et doivent provoquer une usure prématurée de la meule. »

20 L'exploitation de ces carrières est attestée jusqu'au début du XIX ${ }^{\mathrm{e}}$ siècle, $c f$. Michel Martzluff, «Les techniques primitives... ». Les meuniers du département des Pyrénées-Orientales, se fournissent 
moins chères, mais impropres à produire les farines les plus appréciées, les plus fines.

Il faut ici introduire un élément supplémentaire, qui n'est pas seulement l'usure, mais dont l'usure peut être un indicateur : toute meule n'est pas équivalente pour la qualité de la farine produite. La finesse du grain de la pierre de meule conditionne la finesse de la farine et la qualité du pain qui en est tiré. De même l'usure plus ou moins rapide de la pierre de meule laisse dans la farine plus ou moins de poussière de pierre, abrasive et désagréable. Bref entre la pierre et le pain existe une relation directe.

Le rôle du changement dans le choix des meules dans l'amélioration de la qualité de la farine et donc du pain a été mis en lumière par Alain Belmont. ${ }^{21}$ Notons, à titre de comparaison, que dans le dépouillement systématique de 940 manuels de notaires disposant d'un répertoire, pour la période 1500-1770 rédigés à Grenoble, Alain Belmont a relevé « une soixantaine de baux à prixfait » pour des meules, c'est-à-dire de ventes de meules. ${ }^{22}$ Nous avons retrouvé, rappelons-le, 77 actes concernant la vente de 105 meules par Joan Bastard, pour une période de 13 années seulement (1410-1422). Il faut, pour expliquer une telle activité, plusieurs éléments :

1) une forte demande en meules de pierre de Montjuï ;

2) une offre de meules adaptée au marché avec la possibilité de paiement à crédit systématique.

On peut interpréter la demande comme une demande sous-tendue par une exigence sociale de pain de meilleure qualité, assez forte pour pousser les meuniers à renouveler, sans doute au rythme de l'usure de leurs meules tournantes, mais parfois en devançant même cette usure, car de nombreux achats se font pour 2 meules, voire 3, 4 et 5 meules, en une seule fois. Cela ne signifie pas que les meules anciennes, en granite sans doute ou dans un grès moins fin (celui des Molars du Boulou), étaient détruites ou perdues, car dans un casal de deux roues, on pouvait parfaitement conserver une roue à l'œuvre

encore, disent-ils, en meules de granite du Conflent, comme beaucoup d'autres meuniers questionnés dans l'enquête de 1808-1809, mais ils utilisent aussi plus rarement des meules importées de Bordeaux, sans aucun doute pour des moutures plus raffinées. Je n'ai malheureusement pas pu consulter ces enquêtes, citées dans l'article en ligne : https://fr.wikipedia.org/wiki/Meule_à_grains

21 Alain Belmont, « La pierre et le pain. Les carrières de meules de moulin de Quaix-en-Chartreuse (XVI ${ }^{\mathrm{e}}$-XVIII ${ }^{\mathrm{e}}$ siècle) », Histoire et Sociétés Rurales, 2001/2, vol. 16, p. 45-79.

22 Alain Belmont, « La pierre et le pain... », p. 56. 
avec des meules de pierre locale plus grossière, pour des farines de qualité inférieure (seigle, méteil) à côté d'un moulin faisant tourner une des nouvelles meules en grès de Montjuïc, pour une farine blanche de froment de qualité supérieure. Les anciennes meules démontées pouvaient parfaitement être conservées pour servir au remplacement des meules rustiques sur le second moulin.

Si l'on se reporte aux lieux où sont installées ces nouvelles meules, on y trouve bien entendu Perpignan avec 24 actes, les bourgs et villages du Roussillon (Elne : 9 actes ; Millas 5 actes ; Thuir, Le Boulou, Ille : 3 actes ; Canet, Torreilles : 2 actes ; Saint-Estève, Nidolères, Pollestres, Pézilla-laRivière, Calce, Salses, Palau-del-Vidre, Laroque, Boule d'Amont, Bouleternère, Corneilla-de-la-Rivière), mais aussi du Conflent, où l'approvisionnement en meules de granite n'a pas cessé d'exister (Vinça : 5 actes ; Joch : 2 actes ; Marquixanes, Prades), mais est plus rare en Vallespir (seulement deux actes pour Céret et Reynès). Il ne fait pas de doute donc que Joan Bastard est connu partout en Roussillon, Vallespir et Conflent, au moins jusqu'à Prades, comme importateur et marchand à crédit de meules en pierre de Montjuïc.

La demande est forte, et à mon sens, elle est une demande sociale, au sens où de nombreux meuniers souhaitent avoir ces meules, car la farine qu'elles fournissent est devenue une exigence de la clientèle. Pour y répondre vite, Joan Bastard a construit un réseau d'approvisionnement, avec des contrats avec les tailleurs de pierre barcelonais, avec les armateurs qui fournissent les navires et éventuellement les financiers qui ont investi dans l'affaire.

L'étude des relations familiales et sociales de Joan Bastard, de ses autres activités commerciales (essentiellement autour de la vente de céréales, qui lui sont parfois données en paiement des meules), possible grâce aux dizaines de documents réunis, reste à faire, une remarque cependant Joan Bastard et son frère Jaume, qui le remplace parfois devant le notaire pour l'enregistrement d'une vente ou d'un paiement, sont dits tous deux 'forgerons' ils sont les fils d'un Jaume Bastard forgeron, déjà décédé en 1411. Mais tous les actes concernant spécifiquement la vente de produits métalliques ou d'outils de forgeron sont le fait du frère (aîné) Jaume. Joan n'apparaît que dans les ventes de meules et des activités commerciales... Peut-être est-il aussi à l'occasion un forgeron, dans l'atelier de son frère, ou bien son statut social d'artisan lui permet-il d'être intégré à une corporation, même s'il semble davantage être un mercader qu' un fabre, mais il faut supposer que la corporation des mercaders ne devait pas être ouverte à tous... 


\section{Conclusion}

L'hypothèse que j'ai avancée peut sembler fragile, car la durée supposée de vie d'une meule est de 10 à 15 ans. ${ }^{23}$ L'achat de meules de remplacement est donc régulier, et une dizaine ou une vingtaine de meules vendues par an peuvent correspondre au rythme de renouvellement des meules d'une ou deux centaines de moulins fonctionnant en Roussillon au début du $\mathrm{XV}^{\mathrm{e}}$ siècle. Restent cependant quelques éléments d'indices que l'on ne peut écarter : rien dans la documentation de la fin du XIV ${ }^{\mathrm{e}}$ siècle ou de la première décennie du $\mathrm{XV}^{\mathrm{e}}$ ne montre ce flux régulier de renouvellement des meules. Pourquoi ? À mon avis parce que l'achat de meules à des producteurs locaux se fait, dans la plus grande partie des cas, avec un paiement au comptant et sans acte notarié. «L'effet de source » que l'on constate ici est lié à la vente à crédit systématique, qui génère une documentation notariée obligatoire. Mais la production traditionnelle de meules de grès ou de granite ne cesse pas en Roussillon et en Conflent (Le Boulou, Reglella, Rodès), avec la vente directe au comptant ne cesse pas, comme le montre l'étude des carrières d'extraction et elle se maintient, comme le disent les meuniers, jusqu'au début du XIX ${ }^{\mathrm{e}}$ siècle. Donc Joan Bastard a non seulement réussi à drainer une bonne part de l'achat des meules en Roussillon, mais aussi à susciter des achats qui vont au-delà du renouvellement naturel des meules usées. Grâce à sa stratégie de vente à crédit, grâce à un réseau d'approvisionnement en meules bien construit, Joan Bastard permet à des meuniers de s'équiper en meules nouvelles « dernier cri » celles que l'on veut pour pouvoir offrir une belle farine, pour un bon pain de qualité, comme une partie de la population aime à le manger. Après la crise économique et démographique du milieu du XIV siècle, les indicateurs économiques de la reprise de l'économie, et d'une prospérité nouvelle en Catalogne, et à Perpignan en particulier, ${ }^{24}$ sont clairs. Des goûts plus raffinés, une consommation alimentaire plus soignée, comme de meilleurs vins, des plats plus sophistiqués, très épicés, et bien entendu un beau pain blanc, voilà ce qu'une partie enrichie de la population désire. Joan Bastard, ingénieux importateur et négociant, va permettre, en une douzaine d'années, à une grande partie des meuniers du Roussillon de répondre à cette demande sociale.

23 S. Caucanas, Moulins et irrigation en Roussillon..., p. 149.

24 Damien Coulon, Partie II, Moyen Âge, Chapitre 3, « Crises et reprises 1344-1462 », Patrice Poujade (dir.), Histoire de Perpignan, en préparation. 


\section{Références bibliographiques}

BELMONT, Alain (2001) «La pierre et le pain : Les carrières de meules de moulin de Quaix-en-Chartreuse (XVI ${ }^{\mathrm{e}}$-XVIII ${ }^{\mathrm{e}}$ siècle) », Histoire et Sociétés Rurales, 2001/2, vol. $16: 45-79$.

CATAFAu, Aymat, Nikolas JASPERT, Thomas WetzSTEIN (2018), Perpignan 1415. Un sommet européen à l'époque du Grand Schisme d'Occident. Zurich, LIT Verlag.

CAUCANAS, Sylvie (1995) Moulins et irrigation en Roussillon du IXe au XV siècle. Paris, CNRS Histoire.

Coulon, Damien (en préparation), « Crises et reprises, 1344-1462». Dans Patrice Poujade (dir.), Histoire de Perpignan.

GIRONElla I GRANÉS, Josep Maria (2010) Els molins i les salines de Castelló d'Empúries al segle XIV. Barcelona, Fundació Noguera, col. Estudis, 57.

MARTZLUFF, Michel (2009), «II.1. Les techniques primitives : extraction et façonnage opportunistes des meules en granit », dans Olivier Passarrius, Aymat Catafau et Michel Martzluff (dir.), Archéologie d'une montagne brûlée. Perpignan, Trabucaire: $315-319$.

MARTZlufF, Michel et alii (2008), « Meules et moulins de Vilarnau ». Dans Olivier Passarrius, Richard Donat et Aymat Catafau (dir.), Vilarnau. Un village du Moyen Âge en Roussillon. Perpignan, Trabucaire: 314-384. 
